\title{
A brief, highly selective history of acute phase proteins as indicators of infection, inflammation and injury
}

\author{
Michael C. Powanda ${ }^{1}$ [ $\cdot$ Elizabeth D. Moyer ${ }^{1}$
}

Received: 3 May 2021 / Accepted: 9 May 2021 / Published online: 21 May 2021

(c) The Author(s), under exclusive licence to Springer Nature Switzerland AG 2021

\begin{abstract}
There is an array of plasma protein alterations that occur in a wide variety of species, including humans in response to trauma, inflammation and infections, seemingly irrespective of etiologic agent. In numerous species, these plasma proteins are part of the innate immune response. In addition, it appears that a number of the plasma proteins in this array can be predictive of morbidity and/or mortality. We propose that based on historic use, selected acute phase proteins should be included in ongoing and future non-clinical and clinical studies to help us better understand disease progression in chronic, as well as acute diseases. In addition to assess if there is a relationship between vaccine-induced inflammation and degree of protection from live, attenuated or synthetic vaccines.
\end{abstract}

Keywords Acute phase proteins $\cdot$ Animal models of disease $\cdot$ Chronic disease $\cdot$ Covid-19 $\cdot$ C-reactive protein $\cdot$ Innate immunity $\cdot$ Morbidity and mortality $\cdot$ Trauma and sepsis $\cdot$ Vaccination

\section{The present value of C-reactive protein as an indicator of the presence and severity of infection.}

C-reactive protein (CRP) has long been a highly sensitive indicator of the presence and severity of infection, as well as efficacy of treatment in patients. A high-sensitivity C-reactive protein (hs-CRP) test, which is more sensitive than a standard test, also can be used to evaluate the risk of developing coronary artery disease.

In a recent study of 209 patients' findings show that only C-reactive protein (CRP), in the multivariate analysis, was significantly associated with the progression to a severe case of COVID-19 (OR, 1.056; 95\% CI, 1.025-1.089; $p<0.001$ ). The data suggested that for every 1-unit increase in CRP level, the risk of developing severe events increased by about 5\%. (Wang G, Wu C, Zhang Q, et al. (2020). This is consistent with the results of a review of 1896 survivors and 849 non-survivors cases of Covid-19 Sahu et al. (2020). In conjunction with eosinopenia, CRP can help triage patient patients presenting with fevers, sorting suspected COVID-19

Michael C. Powanda

powanda@mpbiomed.com

1 M/P Biomedical Consultants LLC, 402 Live Oak Drive, Mill Valley, CA 94941, USA patients from those with COVID-19-like symptoms. Lia et al. (2020).

\section{The potential of proteomics in disease diagnosis and therapeutic monitoring}

As the authors of a review on human plasma proteins state, "The human plasma proteome holds the promise of a revolution in disease diagnosis and therapeutic monitoring provided that major challenges in proteomics and related disciplines can be addressed." (Anderson \& Anderson, 2002). The authors list 289 proteins whose detection in plasma or serum is documented in the literature. These include enzymes released from tissue as a result of damage, enzyme inhibitors, a series of apolipoproteins, coagulation factors, complement components, immunoglobulins, cytokines and cytokine inhibitors, hormones, as well as albumin and a series of proteins that decrease or increase in response to inflammation, infection and injury. It is this last group that we will primarily focus on. The proteins in this latter group include albumin and transferrin which decrease during infection, inflammation and injury while alpha-1-acid glycoprotein, alpha-1-antitrypsin, alpha-2-macroglobulin, C-reactive protein, ceruloplasmin, haptoglobin, serum amyloid $\mathrm{A}$ and fibrinogen increase. 


\section{Acute phases proteins possible role in vaccination}

A recent review describes a number of acute phase proteins and their importance in vaccination (Rafaat et al., 2020). These authors discuss in some detail the known and likely function of these proteins, a number of which are associated with response to vaccination. They argue that there is an association between the strength of the acute phase response and vaccination efficacy of key importance to human and veterinary medicine. They cite research in which the absence of interleukin-6, a potent inducer of acute phase proteins, increases the mortality of a live vaccine strain of F. tularensis in mice (Kurtz et al. (2013), unfortunately the plasma protein profile was not evaluated to see which proteins may have been altered.

As a separate part of the review, the authors discuss the acute phase protein response to adjuvants. The effects of adjuvants in rabbits indicated that CRP and fibrinogen expression levels were increased following the administration of adjuvant systems AS01 (monophosphoryl lipid A (MPL) and QS-21), AS03 ( $\alpha$-tocopherol and squalene in an oil-in-water (o/w) emulsion), AS15 (CpG 7909, monophosphoryl lipid, and QS-21) and DTPw (monophosphoryl lipid A) (Leroux-Roels, 2010). CRP levels increased 9-26-fold after injection of AS01, AS03, or AS15. Not all adjuvants cause an increase in inflammatory response (Destexhe et al. (2013) The use of other adjuvants such as aluminum phosphate and aluminum hydroxide did not affect CRP expression levels in the elderly or in the young following a diphtheria-tetanus-poliomyelitistyphoid vaccine (Yousfi et al., 2005). Overall, these studies suggest the acute phase protein response in general, and that of CRP in particular, might be a marker for safety and perhaps efficacy in clinical and non-clinical vaccine trials. It would be interesting to test whether acute phase protein alterations after vaccination correlate with protection and/ or antibody titer.

\section{A non-clinical study of acute phase proteins in naïve and vaccinated rats}

The notion of using acute phase proteins to monitor the response to vaccines, as well as the efficacy of vaccination is not new. Naive rats inoculated ip with a live vaccine strain (LVS) of tularemia (Francisella tularensis) displayed decreases in serum zinc concentration and increases in acute phase proteins relative to increasing dose of microorganism. The vaccine strain, safe at a $4 \log$ dose was lethal at 8 logs. The virulent strain (SCHU S4) was uniformly lethal at a $4 \log$ dose in un-immunized rats. In contrast, rats immunized with $4 \mathrm{logs}$ of the vaccine strain were protected when challenged with 4 or $8 \operatorname{logs}$ of the virulent strain.

At the $4 \log$ challenge dose, all vaccinated animals survived and there were no changes in serum zinc or acute phase concentrations. However, at the $8 \log$ dose, though all animals survived, there were significant changes in serum zinc and acute phase proteins which persisted at least 4 days post challenge. Thus, by monitoring these biomarkers in naïve animals exposed to various dose of candidate vaccines and in immunized animals exposed to a range of doses of the etiologic agent, one may be able to select a dose that optimizes protection while minimizing the reaction to vaccination. (Powanda et al 1975)

It should also be noted that both LVS and SCHU S4 caused pyogranulomas in liver and spleen of nonimmune rats. Nonimmune rats given 10(4) SCHU S4 organisms did not survive beyond $72 \mathrm{~h}$, but immune rats given challenge inoculum of 10(8) SCHU S4 organisms developed lesions and survived. Larger doses of LVS resulted in earlier onset of characteristic hepatitis and splenitis in nonimmune rats. Periportal lymphocytic infiltrates were present in the liver $48 \mathrm{~h}$ after SCHU S4 challenge inoculation of immune rats and $96 \mathrm{~h}$ after inoculation of LVS in nonimmune rats and were associated with intense macrophage aggregation. These changes indicate that the pathogenesis of tularemia is a result of the interdependency of the dose and virulence of the causative agent with the immune status of the host and that cellular immunity has a significant role in the response of the rat to tularemia. (Moe JB et al. (1975). The tissue damage associated with tularemia is related to it being able to infect the liver eliciting the innate immune response which includes changes in acute phase proteins. These data raise the question whether and to what degree is inflammation necessary for a live vaccine to effectively immunize? Is concomitant inflammation necessary for all vaccines, live, attenuated or artificial, such as the newly available mRNA vaccines for Covid-19, to effectively immunize? In some cases, adjuvants themselves can cause inflammation; again is inflammation required for maximum efficacy?

\section{Use of acute phase proteins in testing anti-viral drugs}

Acute phase proteins can also be used to test whether proposed therapies induce inflammation. Polyriboinosinicpolycytidylic acid complexes [poly(I)-poly(C)] are potent inducers of interferon In rodents and rabbits. In primates, however, these compounds arc poor interferon inducers, apparently because the complex is rapidly degraded by plasma nucleases. A modified, nuclease resistant form of 
$\operatorname{poly}(\mathrm{I})-\operatorname{poly}(\mathrm{C})$ has therefore been developed for use in man. This modified poly(ICLC), induces interferon in chimpanzees and rhesus and cynomolgus monkeys. Poly(I).poly(C) itself, in addition to inducing interferon, also causes fever and depressions in plasma zinc A study was conducted in rats to see whether there was a dose effect and whether significant changes in plasma proteins occurred. Repeated dose of 0.3 and $3.0 \mathrm{mg} / \mathrm{kg}$ poly(ICLC) were given over a $48 \mathrm{~h}$ period. Both 0.3 and $3.0 \mathrm{mg} / \mathrm{kg}$ poly(ICLC) caused increases in seromucoid (primarily consisting of alpha-1-acid glycoprotein and haptoglobin) up to five fold at $48 \mathrm{~h}$. A 2 to threefold increase in haptoglobin, and a dose dependent increase in alpha -2-macrofetoprotein. (Powanda et al., 1977).

\section{Some caveats about choice of models and relevance to humans}

While acute phase proteins may be used to assess the inflammation potential of a drug or vaccine, the animal model chosen will materially affect which plasma proteins are responders. (Watterson et al., 2009). For example, C-reactive protein and serum amyloid $\mathrm{A}$ are major reactants in humans, while in rats, it is alpha-2-macroglobulin. Both humans and rats have alpha-1-acid glycoprotein, haptoglobin and fibrinogen as moderate reactants, as does dog and mouse. (Watterson et al., (2009) This difference in major and moderate reactants has been observed in other species (Cray C, Zaias J, Altman NH (2009).

It should be noted that acute phase protein synthesis can occur not only in the liver, but also leukocytes, lymph nodes and spleen in a porcine model of infection. (Skovogaard K et. al (2009) Even in healthy chickens mRNA coding for alpha1-acid glycoprotein (AGP), serum amyloid A (SAA), PIT54, C-Reactive protein (CRP) and Ovotransferrin (OVT) could be found in multiple extra hepatic tissues. The mRNA coding for CRP, OVT and SAA was detected in all 20 analyzed tissues with a higher expression in gastrointestinal tract, respiratory and lymphatic samples. ACP positive cells were present in the epithelium of the mucosal layer of gastrointestinal tract and kidney (Marques et al., 2017). These data suggest that with so many cell types containing mRNA for these acute phase proteins, the innate immune response to inflammation, infection and injury could be localized, as well as a systemic.

Though CRP in itself is a good marker for the presence and severity of disease, in a porcine model challenged with one of three bacterial agents, one parasitic and one viral agent, the use of a combination of acute phase proteins allowed the detection of disease with more sensitivity than any individual acute phase protein. (Heegard PMH et. al (2011).

\section{Use of multiple acute phase proteins in monitoring trauma-septic patients}

In a study of trauma-septic patients indicated that select plasma proteins, $\alpha 1$-acid glycoprotein and ceruloplasmin, along with some amino acids and catabolites, allowed one to identify, up to 9 days before their demise, patients who would not survive with $99 \%$ certainty from a single plasma analysis profile (Moyer et al., 1981). There is a reprioritization of hepatic plasma protein release in trauma and sepsis wherein trauma patients who become septic have C-reactive protein, fibrinogen, ceruloplasmin, and $\alpha 1$-antitrypsin levels that continued to be elevated after the initial five posttrauma days, while transferrin, albumin, and $\alpha 2$-macroglobulin levels fell. This reprioritization response seems to be both a predictor of sepsis as well as a measure of the adequacy of the host response to trauma and sepsis (Sganga et al., 1985).

A small study of plasma protein changes in burned patients with and without the complication of infection indicated that the ratios of select plasma proteins, rather than the absolute values, provided a more effective discrimination among those patients who were burned (B) versus those with infection (BI) and versus those who were not only burned and infected, but also had complications requiring ventilatory support and/or some degree of renal impairment (BIC). Stepwise discriminant analysis indicated that the $\mathrm{B}, \mathrm{BI}$ and $\mathrm{BIC}$ patients could be differentiated on the basis of $\alpha 1$-acid glycoprotein (AG) and haptoglobin $(\mathrm{Hp})$ concentration in combination. When the data were rank ordered and subjected to analysis of variance, the ratios of $\mathrm{AG} / \mathrm{Hp}$ and $\mathrm{AG} /$ transferrin were found not only to discriminate $\mathrm{B}$ from BI patients, but also BIC from BI patients. (Powanda, MC, Moyer ED, Wilmore D et al. (1983); Moyer ED, Powanda MC (2004).

Many of these acute phase proteins appear to have significant roles in wound healing. Alpha-1-antitrypsin and alpha-2-macroglobin accumulate at the site of injury presumably to prevent additional damage due to proteolytic enzymes released from already damaged tissue, as well as from phagocytic cells. Alpha-1-acid glycoprotein due to its high-carbohydrate content may act as an adhesive at the wound site. It also can inhibit phagocytosis and lymphocyte transformation and thus could protect the healing wound against the development of auto immunity. Ceruloplasmin transfers copper to cytochrome $\mathrm{C}$ oxidase vital to aerobic energy production, which along with glycolysis increases during wound healing. Ceruloplasmin and the copper it carries are also essential to collagen formation. Haptoglobin increases four to five times its normal plasma concentration following injury and is frequently found in inflammatory exudates. The principal biologic function of 
this protein appears to be the removal of free hemoglobin released by hemolysis. (Powanda and Moyer, 1981); Janciauskiene and T, Mahadeva R, 2011).

\section{Acute phase proteins as a monitor of chronic disease}

Might plasma proteins act as a biological dosimeter for cumulative stress, repeated infection, injury or inflammation and/or as an indicator of physiologic age? Data on the levels of acute phase proteins and plasma protein synthesis rates in adult vs. old rats (Papet et al., 2003) may prove instructive. Older rats (22 months) have increased levels of total proteins, even though albumin concentrations are lower, than adult ( 8 month) rats. There is also a doubling of fibrinogen, a 4.5 -fold increase in $\alpha 2$-macroglobulin and a $35 \%$ increase in $\alpha 1$-acid glycoprotein concentration. There also is an increase in both the fractional and absolute synthesis rates for plasma proteins in the old rats, including for albumin, despite the decrease in its absolute concentration. Thymus weight decreased and spleen weight increased in old versus adult rats. These data are consistent with the "inflamm-aging" concept. (Franceschi et al., 2000).

Age and sex appear to profoundly affect plasma protein concentrations, at least in Gottigen minipigs. Blood samples were taken at 6, 16, 24 and 40-48 weeks. Significant changes by sex $\mathrm{x}$ age were seen in CRP, haptoglobin, pig major acute phase protein (PMAP) and albumin. Porcine $\alpha-1$ acid glycoprotein (PAGP) displayed highly significant $(p<0.001)$ effects of age and gender. CRP, haptoglobin, pig major acute phase protein generally decreased rather than increased with age. Albumin increased with age. (Christoffersen et al., 2015) Changing housing conditions (not specified) caused PAGP, CRP, PMAP and haptoglobin to increase $(p<0.05)$ and albumin to decrease $(p<0.05)$ Are the differences seen in this study versus that of (Papet et al., 2003) due to a difference in species, rat versus minipig? If so, which species better mimics the human response to age?

\section{Summary}

To sum up, despite their past use in non-clinical studies in a variety of species with various infectious and inflammatory agents, and in clinical studies, to predict morbidity and mortality, (Powanda MC, Beisel WR (2003) most acute phase proteins today are not extensively monitored. Considering the role of many of these plasma proteins in the innate immune response in numerous species, this may need reconsideration (Romo MR, Perez-Martinez D, Ferrer CC (2016). Perhaps, it is time to reassess whether it may be beneficial if widespread screening of many, if not all, of these proteins should be done in a variety of populations of different ages and defined health status. Were this done the following hypotheses could be tested.

If one, or more than one, plasma protein is used as a biomarker, will that provide a better understanding as to whether the existence and extent of inflammation following vaccination correlates with antibody titer, $\mathrm{T}$ cell mobilization and/ or protection?

Can a constellation of plasma proteins either by absolute value, by ratios or by altered glycosylation be found that will act as a biological dosimeter with regard to cumulative stress, repeated infection, injury or inflammation? Would such biomarkers be of use in monitoring long-term Covid and help sort which patients might benefit from vaccination?

Can a constellation of plasma proteins either by absolute value, by ratios or by altered composition be found that will act as an indicator of physiologic age, as opposed to chronologic age? A comparison with changes in telomere length which alters with age (Shammas MA (2011) would be interesting.

\section{References}

Anderson NL, Anderson NG (2002) The human plasma proteome history character, and diagnostic prospects. Mol Cell Proteomics $1: 845-867$

Christoffersen BO, Jensen SJ, Ludvigsen TP et al (2015) Age- and sexassociated effects on acute-phase proteins in göttingen minipigs. Comp Med 65(4):333-341

Cray C, Zaias J, Altman NH (2009) Acute phase response in animals: A review. Comp Med 59(6):517-526

Destexhe E, Prinsen MK, van Schöll I et al (2013) Evaluation of $\mathrm{C}$-reactive protein as an inflammatory biomarker in rabbits for vaccine nonclinical safety studies. J Pharmacol Toxicol Methods 68:367-373

El Yousfi M, Mercier S, Breuillé D et al (2005) The inflammatory response to vaccination is altered in the elderly. Mech Ageing Dev 126:874-881

Franceschi C, Bonafe M, Valensin S et al (2000) Inflamm-aging. An evolutionary perspective on immunosenescence. Ann. N.Y. Acad Sci 908:244-254

Heegaard PMH, Stockmarr A, Piñeiro M et al (2011) Optimal combinations of acute phase proteins for detecting infectious disease in pigs. Vet Res 42:50

Janciauskiene S, Welte T, Mahadeva R (2011) Acute Phase Proteins: Structure and Function Relationship. Acute Phase Proteins - Regulation and Functions of Acute Phase Proteins Edited by Francisco Veas. https://doi.org/10.5772/18121

Kurtz SL et al (2013) Interleukin-6 is essential for primary resistance to Francisella tularensis live vaccine strain infection. Infect Immun 2:585-597

Leroux-Roels G (2010) Unmet needs in modern vaccinology: Adjuvants to improve the immune response. Vaccine 28:C25-C36

Li Q, Dinga X, Xiab G et al (2020) Research Paper Eosinopenia and elevated C-reactive protein facilitate triage of COVID-19 patients in fever clinic: A retrospective case-control study. EClinicalMedicine. 23:100375 
Marques AT, Nordio L, Lecchi C et al (2017) Widespread extrahepatic expression of acute-phase proteins in healthy chicken (Gallus gallus) tissues. Vet Immunol Immunopathol 190:10-17

Moe JB, Canonico PG, Stookey JL et al (1975) Pathogenesis of tularemia in immune and non-immune rats. Am J Vet Res 36:1505-1510

Moyer ED, Powanda MC (2004) Plasma proteins as indices of physiologic age and cumulative stress, as well as of disease, inflammation, infection and injury. Inflammopharmacology 12:217-221

Moyer E, Cerra F, Chenier R et al (1981) Multiple systems organ failure: VI. Death predictors in the trauma-septic state - the most critical determinants. J Trauma 21:862-869

Papet I, Dardevet D, Sornet C et al (2003) Acute phase protein levels and thymus, spleen and plasma protein synthesis rates differ in adult and old rats. J Nutr 133:215-219

Powanda MC, Beisel WR (2003) Metabolic effects of infection on protein and energy status. J Nutr 133:322S-327S

Powanda MC, Moyer ED (1981) Plasma proteins and wound healing. Surgery, Gynecology \& Obstetrics 153:749-755

Powanda MC, Cockerell GL, Moe JB et al (1975) Induced metabolic sequelae of tularemia in the rat: correlation with tissue damage. Am J Physiol 229:479-483

Powanda MC, Sammons ML, Stephen EL (1977) Systemic metabolic alterations associated with repeated injections of a modified polyriboinosinic-polyribocytidylic acid complex. Antimicrob Agents Chemother 12:602-605

Powanda MC, Moyer ED, Wilmore D et al (1983) Plasma protein ratios as indicators of infection in severely injured patients. Fed Proc $42: 1188$
Rafaat H, Nabil K, Al-Humadi, (2020) Types of acute phase reactants and their importance in vaccination (Review). Biomedical Reports 12:143-152

Romo MR, Perez-Martinez D, Ferrer CC (2016) Innate immunity in vertebrates: an overview. Immunology 148:125-139

Sahu BR, Kampa RK, Padhi A, Panda AK (2020) C-reactive protein: A promising biomarker for poor prognosis in COVID-19 infection. Clin Chim Acta 509:91-94

Sganga G, Siegel JH, Brown G et al (1985) Reprioritization of Hepatic Plasma Protein Release in Trauma and Sepsis Arch Surg 120(2):187-199

Shammas MA (2011) Telomeres, lifestyle, cancer, and aging. Curr Opin Clin Nutr Metab Care 14(1):28-34

Skovgaard K, Mortensen S, Boye M et al (2009) Rapid and widely disseminated acute phase protein response after experimental bacterial infection of pigs. Vet Res 40(3):23

Wang G, Wu C, Zhang Q et al (2020) C reactive protein level may predict the risk of COVID-19 aggravation]. Open Forum Infect Dis. https://doi.org/10.1016/j.eclinm.100375

Watterson C, Lanevschi A, Horner J, Louden C (2009) A comparative analysis of acute-phase proteins as inflammatory biomarkers in preclinical toxicology studies: implications for preclinical to clinical translation. Toxicol Pathol 37:28-33

Publisher's Note Springer Nature remains neutral with regard to jurisdictional claims in published maps and institutional affiliations. 